\title{
Réseaux volent !
}

Dans I'aviation civile, les réseaux en fibres optiques se développent. Pour quelles raisons ? Pour quelles applications ? Avec quels types de fibres optiques ? Quelle connectique ? Quels acteurs ? Éléments de réponses...

$>$ Jean-Michel MUR • Président du Club optique•jm.mur@orange.fr

A ujourd'hui, quelques centaines de mètres voire un ou deux kilomètres de fibres optiques, selon les appareils, soit environ deux millions d'euros par appareil pour les fibres, la connectique et les harnais. Demain, selon divers experts, ce seront 10 à 20 kilomètres de fibres optiques qui innerveront chaque aéronef. Et le chiffre d'affaires réalisé sera d'autant plus intéressant que le marché de l'aviation commerciale pourrait représenter environ 1500 avions par an, pour les vingt prochaines années, d'après Boeing (figure 1).

La raison majeure de ce déploiement des fibres optiques dans les avions vient de la rencontre des qualités intrinsèques de ce média avec les objectifs poursuivis par I'industrie aéronautique. Les quatre objectifs fondamentaux, dans le développement des offres, produits et technologies de l'aviation civile sont la sécurité, le poids, l'aide au personnel de bord et l'occupation des passagers. Ces objectifs se traduisent pour les équipementiers et câbliers par trois grandes orientations que sont l'avion plus électrique, l'avion plus composite et l'accroissement qualitatif des câblages. Nous allons voir pourquoi et comment la fibre optique joue un rôle déterminant dans l'atteinte de ces objectifs. Attachez vos ceintures...
L'avion plus électrique (APE), aussi appelé Fly by wire, vise le développement des commandes électriques en lieu et place des commandes hydrauliques. Cela se traduit par une forte croissance de la puissance électrique embarquée et l'introduction de nouvelles tensions électriques, d'où le développement de nouveaux câbles en cuivre et en fibre optique et de nouveaux connecteurs.

Pour l'histoire, le Concorde a été le premier à exploiter cette technologie dans le domaine du transport commercial. En 1984, l'Airbus A320 devient le premier avion commercial dont les commandes sont entièrement contrôlées par un calculateur. Puis, en 1994, Boeing a suivi à partir du modèle 777. Aujourd'hui, le Boeing 787 Dreamliner est un bon exemple d'utilisation de la fibre optique et d'un bus de données à haut débit grâce au câblage destiné à cette architecture APE fourni par Labinal.

L'avion plus composite (APC) se caractérise par l'utilisation de plus en plus fréquente des matériaux composites, pour des diminutions de poids, dans la construction des appareils comme pour le Boeing 787 Dreamliner ou le futur Airbus $A 350$ XWB qui devrait arriver fin 2013. Cela induit des contraintes plus sévères aux niveaux thermiques et risque de foudre sur le câblage. Mais, surtout, le fait que la carlingue ne fasse plus cage de Faraday limite fortement l'emploi des émissions radio type Wi-Fi et oblige les câbles en cuivre à être écrantés, blindés et surtressés, synonyme de surplus de poids et de perte de souplesse. En effet, le risque est grand que les interférences électromagnétiques (IEM) et les interférences radioélectriques produites par les équipements personnels des passagers - téléphones mobiles, tablettes informatiques, consoles de jeux, etc. - interviennent sur d'autres appareils de bord ou au sol. Ainsi, pour des raisons de sécurité et comme avantage-clé son insensibilité aux IEM, la fibre optique gagne du terrain sur ces applications.

Il est intéressant de noter que les connecticiens suivent cette tendance APC. Par exemple, Amphenol Air LB commercialise le SIM Mono Module, élément en composite pouvant recevoir aussi bien de la puissance que du signal sur cuivre ou de la fibre optique.

L'accroissement qualitatif des câblages se retrouve dans les améliorations constantes de l'aide au personnel de bord et du confort du passager, domaine de prédilection pour la fibre optique (figure 2).

Figure 1. Perspectives du marché mondial des avions, de 2009 à 2028, toutes marques confondues.

\begin{tabular}{|c|c|c|c|c|c|}
\hline \multicolumn{3}{|c|}{ Avions en service 2008 at 2028} & \multicolumn{3}{|c|}{ Demande par type 2009 à 2028} \\
\hline Type & 2008 & 2028 & Type & Nouveaux avions & Valeur en milliards de $\$$ \\
\hline Gros porteurs & 870 & 1070 & Gros porteurs & 740 & 220 \\
\hline Bicouloirs & 3510 & 8080 & Bicouloirs & 6700 & 1510 \\
\hline Monocouloirs & 11360 & 24230 & Monocouloirs & 19460 & 1420 \\
\hline Jets régionaux & 3060 & 2220 & Jets régionaux & 2100 & 70 \\
\hline Totaux & 18600 & 35600 & Totaux & 29000 & 3220 \\
\hline
\end{tabular}

Source : site internet de Boeing France, mars 2011, http://www.boeing.fr/Redirect.do?Keyword=cmo_2009_1-1 


\begin{tabular}{|c|c|c|c|c|}
\hline Aircraft family... & A320 & $\begin{array}{l}\text { A330 } \\
\text { A340 }\end{array}$ & A380 & A350 \\
\hline \multicolumn{5}{|l|}{ Optical fiber application in Airbus aircraft } \\
\hline CDS - Cockpit display system & & & * & \\
\hline Large displays (under development) & & & & * \\
\hline HUD - Head-up display & * & * & $*$ & * \\
\hline OANS - On-board airport navigation system & * & * & $*$ & * \\
\hline TACS - Taxi aid camera system & & * & $*$ & * \\
\hline CMV - Concentrator multiplexing video & & & * & \\
\hline $\begin{array}{l}\text { NSS/OIS - Network server system / } \\
\text { On-board information system }\end{array}$ & & & & * \\
\hline CVMS - Cabin video monitoring system & & & * & \\
\hline CDSS - Cockpit door surveillance system & & & * & \\
\hline IFE - In-flight entertainment & & * & $*$ & * \\
\hline \multicolumn{5}{|l|}{$\begin{array}{l}\text { Figures (average following chosen options) - } \\
\mathrm{TBD}=\text { to be determined }\end{array}$} \\
\hline Cable length & \multirow{2}{*}{$\begin{array}{l}\text { following } \\
\text { chosen } \\
\text { options }\end{array}$} & $565 \mathrm{~m}$ & $2,4 \mathrm{~km}$ & TBD \\
\hline Number of links & & 41 & 171 & TBD \\
\hline
\end{tabular}

Figure 2. Panorama des applications de la fibre optique dans les avions Airbus.

L'aide au personnel de bord évolue avec les applications recouvertes par l'acronyme IFN - In-flight network. En exem- ples d'IFN, on note le transfert de fichiers importants pour des applications concernant les opérations d'assurance de la qualité (Flight operations quality assurance - FOQA) ou celles concernant la documentation avec la sacoche de vol électronique (Electronic flight bag EFB). À ce sujet, Radiall a été sélectionné par Boeing dans la catégorie « Common Aerospace Commodities " et fournit pour le 787 Dreamliner un vaste portefeuille de produits de connectique, dont les systèmes de connexion par fibre optique pour l'EFB.

Les améliorations pour les passagers sont les applications qui, hier, concernaient le loisir pur et étaient appelées In-flight entertainment (IFE). C'était, par exemple, la possibilité d'écouter de la musique ou de choisir un film et le regarder à sa place dans la langue de son choix, avec ou sans texte incrusté dans l'écran, applications type audio-video on demand (AVOD) ou bien avoir accès à des jeux (In-flight games), cela sur un écran de télévision personnel. À titre d'exemple, lors du salon Aircraft Interior Expo de Hambourg (5 au 7 avril 2011), Lumexis Corp. présentait son système

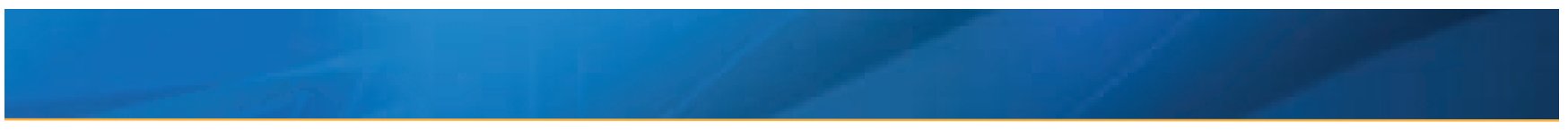

\section{Solutions complètes pour contrôler vos diodes lasers}

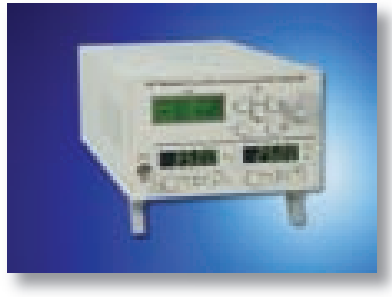

Contrôleurs LD/TEC

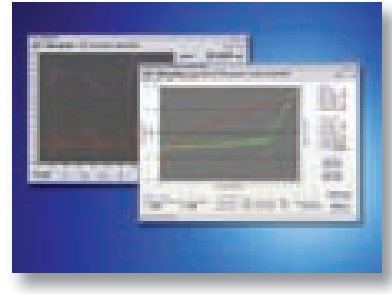

Suite de logiciels

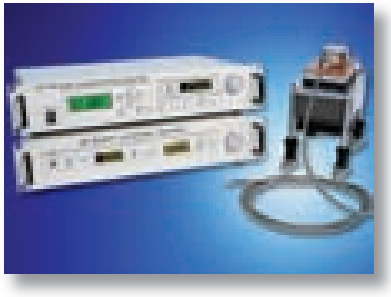

Kits de contrôle de diodes lase

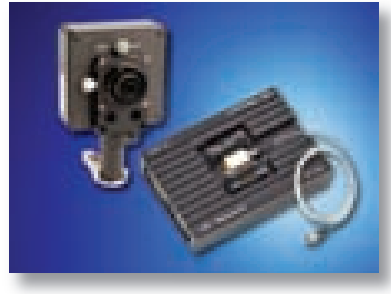

Montures laser

Depuis de nombreuses années, les instruments de contrôle de diode laser Newport servent de nombreuses applications clients dans divers domaines scientifiques et industriels. Aujourd'hui nous offrons de nouvelles solutions complètes de contrôle de diodes lasers, encore plus performantes, innovantes et très économiques. Pour en savoir plus, visitez la page www.newport.com/ld-control-5.

Inscrivez en ligne pour recevoir en priorité le prochain catalogue général MICRO-CONTROLE 2011/2012 www.newport.com/resource2011

MICRO-CONTROLE Spectra-Physics S.A.S

1, rue Jules Guesde - Bâtiment B Tél.: 01.60.91.68.68

Zl. du Bois de l'Épine - BP189 Fax: 01.60.91.68.69

91006 Évry CEDEX e-mail : france@newport.com

\section{MICRO-CONTROLE}

une marque de Newport Corporation

C 2011 Newport Corporation

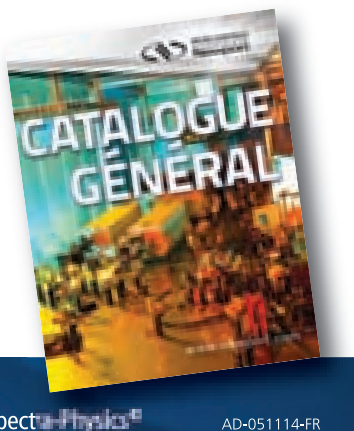

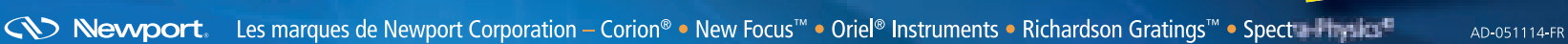


«Fiber-to-the-screen » amenant à chaque siège les applications audio-vidéo à la demande avec la télévision en haute définition. En plus, aujourd'hui, ce qui est recherché, ce sont les applications de communication ou d'information dénommées In-flight connectivity (IFC) avec le souhait d'envoi et réception de SMS, de connexion sur Internet à partir de son micro-ordinateur ou tablette personnelle, de connaissance des conditions climatiques à l'arrivée, etc. Cette évolution se retrouve dans les offres regroupées sous le nom In-flight entertainment and connectivity (IFEC). En acteurs, signaIons Thales, avec son système TopSeries IFE et son TouchPMU (passenger media unit) qui a été distingué «2010 Avion award for best in technology", et Carlisle Interconnect Technology avec sa dernière offre de mars 2011, LITEflight Optical HD, destinée aux jets d'affaires équipés d'écrans HD plasma ou à DEL.

Enfin, ces avantages de poids et de souplesse signifient soit plus de fret à emporter, soit moins de kérosène à consommer. Également, l'encombrement beaucoup plus réduit d'un câble en fibre optique et la souplesse dans le montage et le cheminement permettent des gains complémentaires comme dans l'installation des sièges qui, de plus, pourraient être plus fins.

\section{Fibres et câbles}

Cela étant, quelles sont les fibres optiques rencontrées dans les avions ? Les premières ont été des fibres optiques multimodales à gros cœurs de $200 \mu \mathrm{m}$ et de $230 \mu$ m de gaine-les modèles $200 / 230 \mu \mathrm{m}$ - et celles ayant un cœur de $100 \mu \mathrm{m}$ avec une gaine de $140 \mu \mathrm{m}$ - les 100/140 $\mu \mathrm{m}$. L'avantage majeur résidait dans la facilité de montage et donc de maintenance sur les aéroports, où qu'ils soient situés, d'autant que les débits nécessaires aux applications d'alors étaient suffisants. Mais, peu à peu, comme pour les réseaux d'entreprises sur «le plancher des vaches", ces fibres ont été remplacées par les fibres multimodales de cœur et de gaine plus fins, les fibres $62,5 / 125 \mu \mathrm{m}$ et les 50/125 $\mu \mathrm{m}$. Une gaine plus fine permet de gagner en volume occupé mais, surtout, un cœur plus fin autorise des débits plus élevés. Cette montée en débits est similaire à celle rencontrée en entreprise avec des applications s'appuyant sur diverses versions du protocole Ethernet : le Fast Ethernet à $100 \mathrm{Mbit} / \mathrm{s}$, I'Ethernet commuté comme I'Avionics full duplex switched Ethernet ou AFDX (voir encadré) et le Gigabit Ethernet (GbE). C'est ainsi que la décennie 2000 a vu l'installation des fibres $62,5 / 125$ dotées d'une bande passante de 160 à $300 \mathrm{MHz} . \mathrm{km}$ et, pour la décennie 2010, la tendance est aux fibres 50/125 aux bandes passantes de 500 à $1000 \mathrm{MHz}$.km, voire davantage pour certaines offres. Les évolutions des réseaux d'entreprises vers le $10 \mathrm{GbE}$ et les études en cours laissent à penser que les fibres optiques unimodales de $9 \mu \mathrm{m}$ de cœur et $125 \mu \mathrm{m}$ de gaine seront installées à terme. À ce titre, il sera intéressant de suivre les résultats du programme de recherche européen Daphné (voir encadré).

Mais le problème majeur rencontré dans le câblage n'est pas la fibre elle-même : l'accouplement fibre-câble est au centre du débat. En effet, en réseau d'entreprise, il y a deux types de structure de câble. On trouve la structure serrée, où câble et fibre sont solidaires, et la structure lâche, où la fibre peut «jouer » dans le câble. La raison majeure vient du dif- férentiel des coefficients de dilatation et/ou rétractation qui sont différents entre la fibre en silice et le câble. Les domaines d'utilisation sont clairs. La structure serrée est employée à l'intérieur des bâtiments et la structure lâche est usitée en extérieur. Dans les avions, il ne peut pas y avoir de structure lâche à cause des accélérations et décélérations mais aussi des vibrations qui pourraient entraîner des tensions sur les fibres et des écrasements sur les faces de contact. Aussi, on rencontre des structures serrées dont la difficulté majeure est la constitution du câble-tendance en Europe - et des structures semi-lâches, plutôt aux États-Unis.

Comme autres difficultés techniques, on rencontre la tenue aux agressions chimiques des fluides; la gamme de températures à supporter qui va de $-55^{\circ} \mathrm{C}$ à $+125^{\circ} \mathrm{C}$ et même $155^{\circ} \mathrm{C}$; la performance mécanique et, surtout, la tenue mécanique longitudinale qui sont traitées par des tresses d'aramide ou de fibres de verre ; le respect de la réglementation zéro-halogène, etc. Côté normalisation, les câbles les plus fréquents ont un diamètre extérieur de $1,8 \mathrm{~mm}$ et coexistent avec des câbles de 2,76 mm, essentiellement pour les interconnexions, et, parfois, plus de $5 \mathrm{~mm}$. En illustration (figure 3), I'offre Nexans pour Airbus d'un câble à fibre optique multimodale $62,5 / 125 \mu \mathrm{m}$. La filiale française de Draka, la société

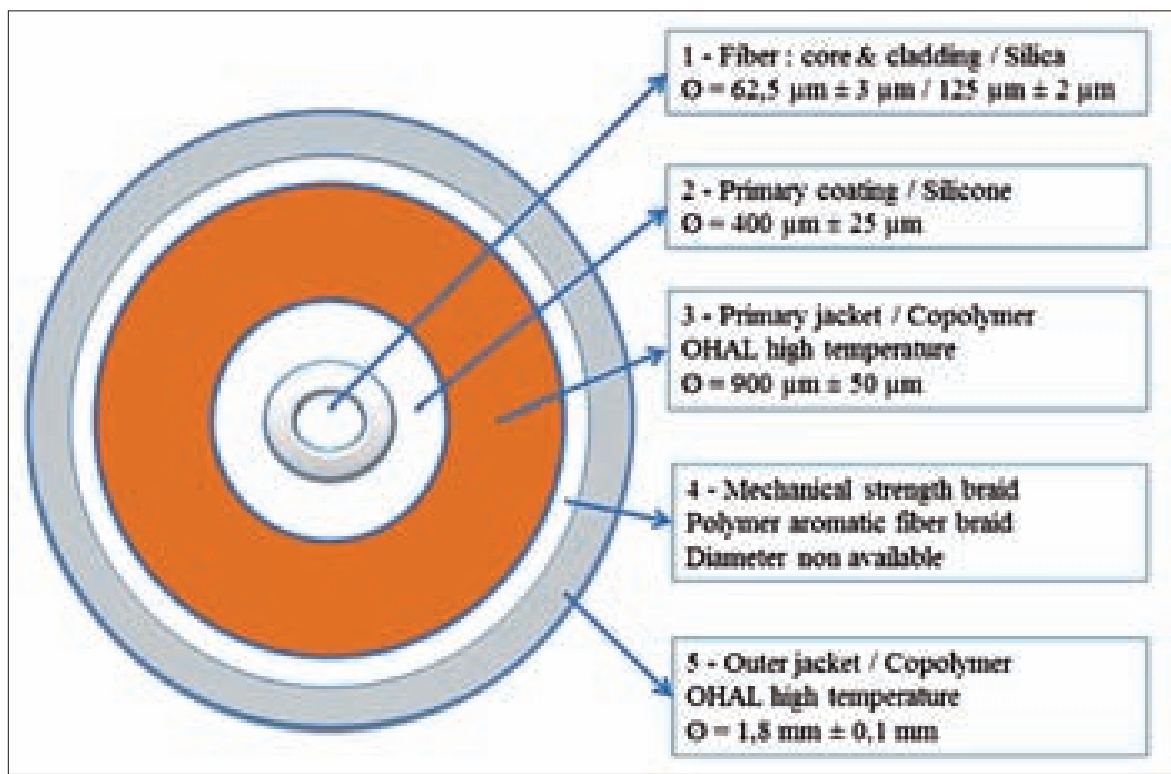

Figure 3. Constitution du câble optique référencé P/N ABS0963-003 LF d'Airbus. 
Draka Fileca située à Sainte-Geneviève, dans l'Oise, propose un catalogue très fourni.

\section{Connectique optique}

Les fibres optiques sont là, mais comment les abouter ? En catimini, sont arrivés les termini... De fait, les normes aéronautiques sont très contraignantes et très difficiles à modifier. Aussi, la connec-

\section{Mais oui, l'Ethernet commuté existe en avionique !}

Parmi les solutions de transmission de données en avionique, signalons I'Avionics full duplex switched Ethernet ou AFDX proposé par Airbus Deustchland $\mathrm{GmbH}$. Il est construit sur la base de nombreux paramètres de l'Ethernet IEEE 802.3 classique tels I'adressage MAC, le protocole IP, le datagramme utilisateur UDP, SNMP... Mais AFDX a un fonctionnement déterministe, requis par les applications avioniques ayant des contraintes de temps réel, et une redondance, pour la sécurité. Il assure la transmission des données aux débits de $10 \mathrm{Mbit} / \mathrm{s}, 100 \mathrm{Mbit} / \mathrm{s}$ et $1 \mathrm{Gbit} / \mathrm{s}$.

L'AFDX est essentiellement une architecture de réseau composée de trois types d'éléments : les liens, en paires torsadées ou fibre optique ; les commutateurs, pour la transmission des paquets de données et la gestion du trafic; et les équipements d'extrémité, recevant ou envoyant des données à travers le réseau. L'échange des trames de données entre équipements

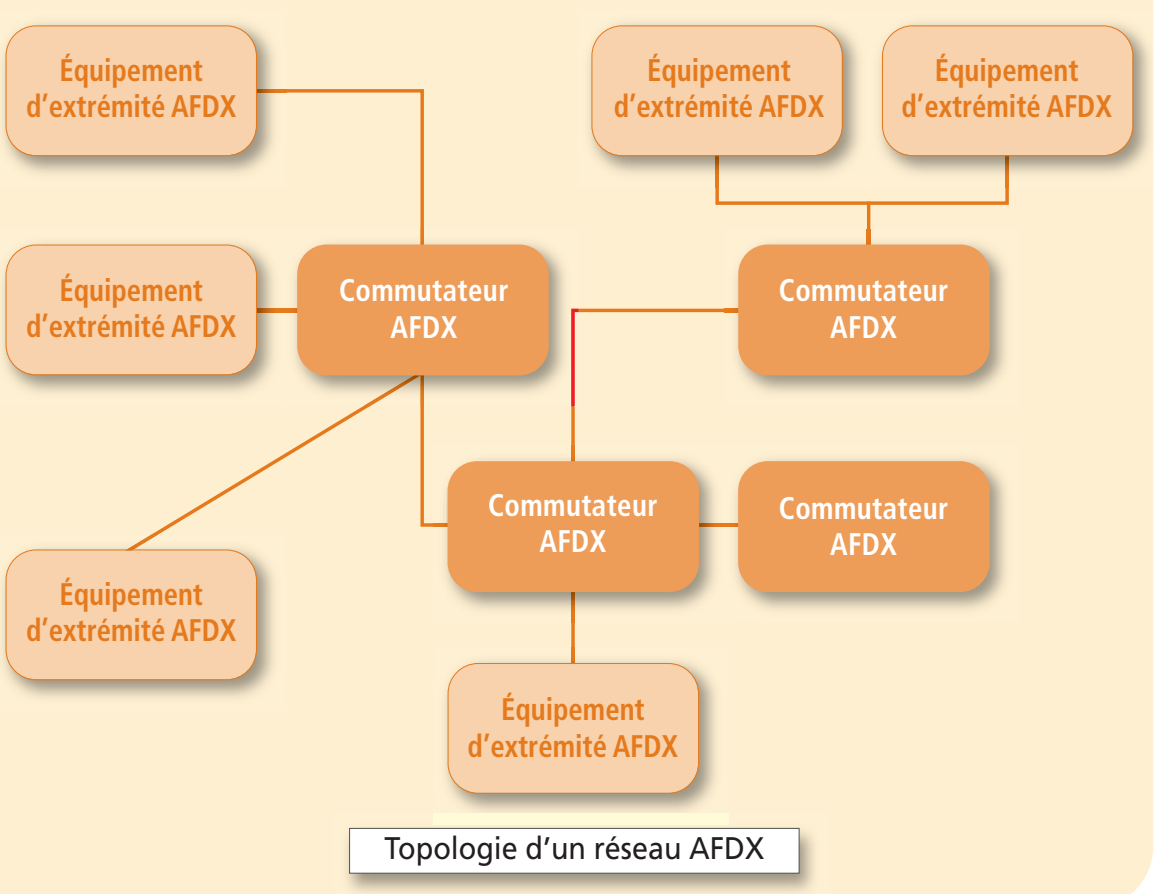

passe par des liens virtuels définissant une connexion logique partant de l'équipement émetteur vers un ou plusieurs équipements destinatairesi.e. communication unitaire (unicast) ou en mode diffusion (multicast). Un réseau AFDX peut définir

Côté sécurité, toutes les connexions sont en duplex intégral sans dorsale dédiée pour les communications entre commutateurs. La redondance est assurée par la duplication des liens et des commutateurs soit, en quelque sorte, l'équivalent de deux réseaux physiques distincts. Les caractéristiques d'AFDX sont en correspondance avec les normes ARINC dont celles concernant les réseaux - Aircraft data network telles que définies dans les parties 2 et 7 de la spécification ARINC 664. Le premier AFDX utilisé en vol l'a été en avril 2005 sur un Airbus $A 380$, prémices d'installation sur d'autres avions - A350, A400M - d'autant que Boeing a été séduit par ce type de réseau pour son B787 Dreamliner. tique optique est arrivée en se fondant dans les connecteurs normalisés à travers un «terminus» (pluriel : des termini), c'est-à-dire un ensemble constitué d'une ferrule et d'un contact. Ainsi, ces termini se sont logés dans des coquilles normalisées, de formes circulaires ou rectangulaires et de tailles diverses, contenant un nombre variable de contacts pour d'autres supports comme des câbles en cuivre. La normalisation permet à $\gg$

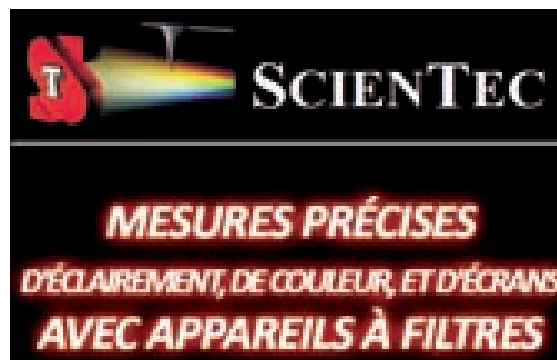
jusqu'à 64000 liens virtuels !

Analyseur d'écrans

LCD, TFT, CRT, PLASMA, FED, OLED DLP

- Mesures en luminance $(\mathrm{cd} / \mathrm{m} / \mathrm{m}$

couleur, temperature de couleut

- Controle qualité en chmmaticite

- Ajustage balance de blane

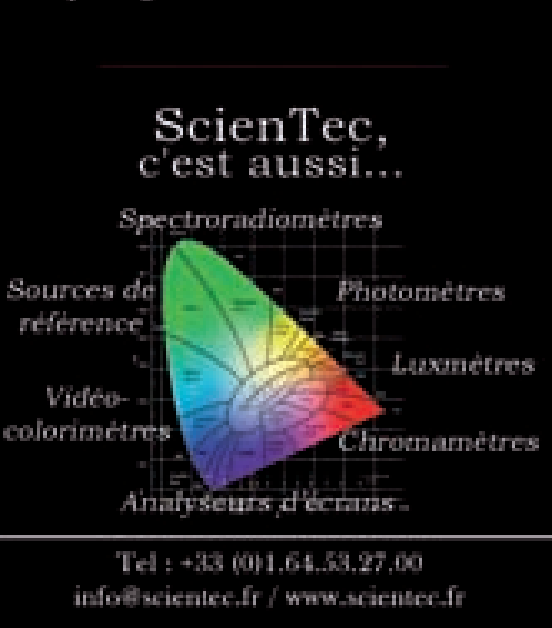


un terminus de se loger dans divers types de coquilles et à une coquille d'accepter divers types de termini. En quelque sorte, le fournisseur construit une interface " géométrique ", cylindrique ou rectangulaire, avec les contacts idoines et trois types de verrouillage : à vis, à baïonnette ou push-pull.

Visitons quelques exemples de termini... Amphenol propose plusieurs types dont MIL-PRF-29504, HD20, ARINC 801 et ferrules MT. Delphi présente une gamme très large y compris des connecteurs optiques hermaphrodites et des cordons optiques pour des applications audio, vidéo et données comme DFOCA (Delphi fiber optic cable assembly). La société Deutsch est présente à travers plusieurs produits dont MC3 pour 5, 8 ou 12 fibres, MC4 pour câbles optiques duplex et le MC5 à haute densité de 2 à 30 voies. Radiall a également une gamme répondant aux principales normes en vigueur en avionique dont sa solution LuxCis, qui plaît beaucoup à Boeing et qui permet I'interconnexion de fibres multimodales et unimodales avec contacts PC et APC ; I'offre a été complétée en juillet 2010 par de nouveaux adaptateurs optiques (size 5 EN4531 optical adapter) pouvant remplacer des contacts coaxiaux. Quant à Souriau, le slogan pour son terminus ELIO est très clair - The same contact in many connector types - et, de fait, ELIO peut être inséré dans les coquilles répondant aux normes Arinc 404, Arinc 600, Arinc 809, EN3646, MIL-DTL-38999/ EN3645... Ainsi, l'aboutement des fibres optiques est réalisable avec un grand nombre de solutions qui doivent répondre à de nombreuses contraintes mécaniques, thermiques, etc. Cependant, parmi les difficultés rencontrées on trouve la poussière en tant qu'ennemi numéro UN et d'autres risques de diminution du signal transporté comme la distance entre les fibres, leur désalignement axial ou leur désalignement angulaire (figures 4), d'où l'importance de la qualité de verrouillage et d'étanchéité.

Dans cet article, seules les parties fibres et connectique ont été présentées. Bien évidemment, pour le bon fonctionnement des réseaux avioniques, il faut ajouter les émetteurs (diodes laser et VCSEL émettant dans les fenêtres de 850 ou $1300 \mathrm{~nm}$ ) et les photodiodes réceptrices, ainsi que divers équipements actifs comme les commutateurs. Trois exemples : 2009, un système de D-Lightsys (filiale de Radiall), en lien avec GigOptix, pour l'Airbus A400M ; octobre 2010, annonce de la gamme Sabre de Protokraft, avec interface optique Elio de Souriau ; mars 2011, offre Hyperion de Teledyne
Reynolds. À ne pas oublier, pour les mesures et la maintenance, les réflectomètres optiques comme le LOR-220 du suisse Luciol Instruments. Les services ne sont pas en reste. À titre d'exemple, depuis le début de l'année 2011, Airbus a développé et propose, dans son catalogue de formation, un cours spécifique dédié à la recherche de pannes et à l'entretien des réseaux en fibres optiques. Enfin, pour ceux qui souhaitent aller plus loin, signalons deux manifestations européennes :

- une au Royaume-Uni, à Swindon, FOHEC - Fibre optics in harsh environnements conference (www.fohec.co.uk) ; - I'autre en Allemagne, à Hambourg, Aircraft Interiors Expo (www.aircraftinteriorsexpo.com). Visites fort enrichissantes garanties...

\section{Connaissez-vous Daphné ?}

Non, il ne s'agit pas de la nymphe aimée d'Apollon, le dieu de la beauté et de la lumière, mais, plus prosaïquement, de I'acronyme Developping Aircraft PHotonic NEtworks.

Daphné est un projet soutenu par la commission européenne FP7 qui a été initié en septembre 2009 pour une durée de trois ans. De manière pragmatique, Daphné a comme objectif d'étudier la transposition des réseaux en fibres optiques terrestres, qu'ils soient pour les télécoms ou industriels, dans les aéronefs européens.

Les principales différences sont la conception des réseaux et leur modèle économique du fait du nombre de nœuds des réseaux, quelques milliers dans les avions mais des millions dans les télécoms, de la taille des liens, quelques mètres et non pas des kilomètres; de la gamme des trafics à supporter, de quelques bits à plusieurs gigabits par seconde, d'autant que certains protocoles avioniques ne sont pas compatibles avec les fibres optiques; des limites phy- siques des composants terrestres qui n'ont pas été conçus pour résister à de larges plages de températures, à des chocs, des vibrations, des tenues au feu, etc.

Ainsi, les principaux défis à relever concernent l'adaptation des technologies des réseaux optiques dans les aéronefs, la définition d'infrastructures modulaires pour ces réseaux avioniques, le développement de composants photoniques supportant les contraintes de l'environnement avionique et, surtout, comment porter la «bonne parole» à l'ensemble des acteurs de l'industrie aéronautique pour la diffusion de ces nouveaux équipements.

Le leadership technique de Daphné a été confié à Nicholas Brownjohn d'Airbus et, parmi les initiateurs du projet, on compte la filiale française de Draka, la société Draka Fileca implantée à SainteGeneviève (Oise).

Plus amples renseignements sur le site internet de Daphné : www.fp7daphne.eu

Figures 4. Pertes dues à la connectique optique
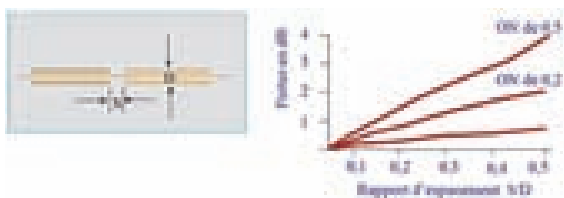

a. Pertes dues à un écartement longitudinal.
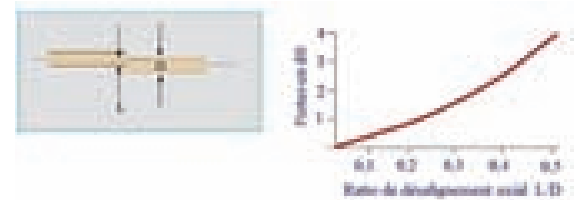

b. Pertes dues à un désalignement axial.
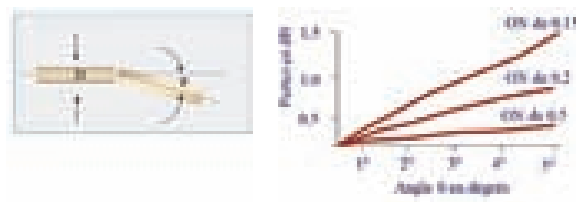

c. Pertes dues à un désalignement angulaire. 